\title{
Relativistic hypernuclear compact stars with calibrated equations of state
}

\author{
Morgane Fortin $\odot^{*}$ \\ N. Copernicus Astronomical Center, Polish Academy of Sciences, Bartycka 18, 00-716 Warszawa, Poland \\ Adriana R. Raduta $0^{\dagger}$ \\ National Institute for Physics and Nuclear Engineering (IFIN-HH), RO-077125 Bucharest, Romania \\ Sidney Avancini $\oplus^{*}$ \\ Departamento de Fisica, Universidade Federal de Santa Catarina, \\ 88040-900 Florianopolis, Santa Catarina, Brazil \\ Constança Providência $\odot^{\S}$ \\ CFisUC, Department of Physics, University of Coimbra, 3004-516 Coimbra, Portugal
}

(Received 6 December 2019; accepted 19 January 2020; published 18 February 2020)

\begin{abstract}
Within the covariant density functional theory of hypernuclear matter we build a series of equations of state for hypernuclear compact stars, by calibrating the coupling constants of the $\Xi$-hyperon to the experimental binding energy of the single- $\Xi$ hypernuclei ${ }_{\Xi}^{15} \mathrm{C}$ and ${ }_{\Xi}^{12} \mathrm{Be}$. Coupling constants of the $\Lambda$ hyperon to nucleons have been calibrated on a vast collection of experimental data on single $\Lambda$ hypernuclei and we employ those values. Uncertainties on the couplings of the $\Sigma$-hyperon to nuclear matter, due to lack of experimental data, are accounted for by allowing for a wide variation of the well depth of $\Sigma$ at rest in symmetric saturated nuclear matter. To account for uncertainties in the nucleonic sector at densities much larger than $n_{0}$, a rich collection of parametrizations is employed, some of them in agreement with existing constraints from nuclear physics and astrophysics. Neutron star properties are investigated with all these calibrated equations of state. The effects of the presence of hyperons on the radius, on the tidal deformability, on the moment of inertia, and on the nucleonic direct Urca process are discussed. The sensitivity of the hyperonic direct Urca processes to uncertainties in the nucleonic and hyperonic sectors is also addressed. It is shown that the relative variations of the radius, tidal deformability and moment of inertia from the values that characterize purely nucleonic stars are linearly correlated with the strangeness fraction. The maximum radius deviation, obtained for most massive neutron stars, is $\approx 10 \%$. The reduction of the maximum mass, triggered by nucleation of strangeness, is estimated at $\approx 15 \%-20 \%$, out of which $5 \%$ comes from insufficient information on the $\Sigma$ hyperon interactions. A total of 44 calibrated hyperonic equations of state are published as Supplemental Material.
\end{abstract}

DOI: 10.1103/PhysRevD.101.034017

\section{INTRODUCTION}

The recent detection of gravitational waves emitted during the inspiral phase of a neutron star-neutron star merger GW170817 [1] together with the following up

\footnotetext{
*fortin@camk.edu.pl

†araduta@nipne.ro

*sidney.avancini@ufsc.br

${ }^{\S} \mathrm{cp} @$ uc.pt
}

Published by the American Physical Society under the terms of the Creative Commons Attribution 4.0 International license. Further distribution of this work must maintain attribution to the author(s) and the published article's title, journal citation, and DOI. Funded by SCOAP ${ }^{3}$. electromagnetic signal opened a new door to the study of neutron stars (NS) [2]. NSs have been acknowledged since long ago to be perfect test grounds of cold and dense baryonic matter, with thermodynamic conditions complementary to those produced in terrestrial laboratories. In the innermost shells non-nucleonic degrees of freedom such as hyperons and kaon or pion condensates or a quark gluon plasma have been predicted to exist [3] in addition to or instead of the nucleonic ones. Understanding the way in which these "exotic" degrees of freedom affect the structure and evolution of NS ultimately allows one to confirm or, on the contrary, rule out their presence. Information thus implicitly gained on the so far insufficiently constrained interaction potentials makes NSs a promising research field for nuclear physics. 
The major source of uncertainties that affect NSs comes from the nucleonic sector, which dominates in all but pure quark stars and whose behavior at densities much larger than the saturation density of symmetric nuclear matter $\left(n_{0}\right)$ and high isospin asymmetry remains poorly known, despite intense theoretical and experimental effort. To account for this state of facts NS studies typically allow for the widest collection of equations of state (EoS) compatible with constraints from nuclear physics experiments, $a b$ initio calculations and astrophysical observations. In the present work we adopt the same strategy. Additional sources of uncertainties are related to the above-mentioned "exotic" species. They are less serious than the ones in the first category, as only NSs with masses exceeding the threshold value for nucleation of those species are affected. Some of the first new degrees of freedom that are expected to be populated are the hyperons, which make the object of our present study. Another possibility is the $\Delta$ resonance [3] which has recently been investigated by several authors [4-7], but which is not considered in the present study.

With the aim of building EoS as realistic as possible, we continue the work started in Refs. [8-10], where the $\Lambda$-nucleon and $\Lambda-\Lambda$ interaction potentials have been constrained based on a vast collection of experimental data on single- and double- $\Lambda$ nuclei, by constraining the $\Xi$-nucleon potentials on experimental data on single- $\Xi$ nuclei. As in Refs. [8-10], we calculate the binding energies of nuclei with a variable number of nucleons and one hyperon by solving the Dirac equations of the nucleons and the hyperon obtained from the assumed Lagrangian density. The coupling constants between the $\Xi$ and the scalar $\sigma$ meson are tuned on the binding energy of ${ }^{15} \mathrm{C}$, measured by the KEK-E373 experiment [11]. The thus obtained EoS are called calibrated, as they comply with the maximum available experimental information. The third hyperonic species expected to nucleate in NS cores is $\Sigma$. Experimental data on strong-interaction level shifts, widths and yields collected from $\Sigma^{-}$atoms and inclusive $\left(\pi^{-}, K^{+}\right)$spectra on medium to heavy targets indicate a repulsive $\Sigma \mathrm{N}$ potential. According to Refs. [12-16], these data are compatible with a wide range of the well depth of $\Sigma$ s at rest in saturated symmetric nuclear matter, $10 \lesssim U_{\Sigma}^{(N)} \lesssim 50 \mathrm{MeV}$. Theoretical studies performed within the chiral effective field theory support the repulsive character of $U_{\Sigma}^{(N)}$, though the magnitude is estimated to lesser values $\approx 15 \mathrm{MeV}$ [17]. Not being able to constrain the couplings of the $\Sigma$-hyperon, we investigate how the uncertainties that affect $U_{\Sigma}^{(N)}$ impact the NS properties. Special attention is given to the chemical composition of NS, particularly sensitive to negatively charged particles and susceptible to being indirectly determined from NSs' thermal evolution. Notable effects are expected to occur for the less repulsive potentials, which favor earlier onset of $\Sigma$ s. This expectation relies on the fact that, as soon as they appear, any negatively charged particles partially replace the electrons in the net charge neutrality equation and, consequently, alter the $\beta$-equilibrium conditions which determine the relative abundances. In extreme scenarios, also the threshold of nucleonic dUrca may be affected.

The first high-precision measurement of a massive pulsar mass, corresponding to PSR J 1614-2230 [18,19] with $M=1.908 \pm 0.016 M_{\odot}$ (in the following masses are given with a precision at the $1-\sigma$ level that is a $68.3 \%$ confidence level), challenged the nuclear physics community on whether two solar mass NSs can accommodate nonnucleonic degrees of freedom $[18,20]$. The particular case of the onset of hyperons, commonly known as the hyperon (ization) puzzle, was addressed at length in Ref. [21], where several scenarios that reconcile large masses and hyperonic degrees of freedom have been identified. They include (i) a sufficiently hard nucleonic EoS and (ii) going beyond the simple $S U(6)$ symmetry ansatz to fix the vector meson couplings [22-28]. Other massive pulsars have been detected in the meanwhile, in particular, the pulsars PSR $\mathrm{J} 0348+0432$ [29], with a mass $2.01 \pm 0.04 M_{\odot}$, and the millisecond pulsar MSP J0740 + 6620 [30], with a mass $2.14_{-0.09}^{+0.10} M_{\odot}$. It is worthwhile to note that massive NSs can be theoretically obtained also by assuming a deconfinement phase transition to quark matter [31-42]. In the present paper we add some more information to the issue and study, within a relativistic mean-field (RMF) approach with model parameters fitted to experimental data, under which conditions NS cores do accommodate hyperons and how these extra particle degrees of freedom modify NSs' geometric and chemical properties. The extent to which one may learn information on chemical composition from thermal evolution is addressed elsewhere.

The paper is organized as follows. Section II presents the nucleonic EoSs on which our hyperonic EoSs are built and the way in which $\Xi$-meson coupling constants are determined from experimental $\Xi$-hypernuclei data. Physical (maximum mass and radius, tidal deformability, and moment of inertia versus gravitational mass) and chemical properties of hypernuclear compact stars built upon our set of calibrated EoSs are discussed in Sec. III. Special attention is given to the uncertainties related to the $\Sigma$ potential. The conclusions are drawn in Sec. IV.

\section{EQUATIONS OF STATE}

In the following we define the set of EoSs that we choose to perform our study. The parametrizations employed to describe the nucleonic sector are introduced in Sec. II A. The way in which experimental data on hypernuclei are used to calibrate the hyperon-meson coupling constants is detailed in Sec. II B. 


\section{A. Nucleonic EoS}

The phenomenological EoS considered in our study have been obtained in the framework of RMF. In this category of models the nucleons interact among each others by exchanging scalar-isoscalar $(\sigma)$, vector-isoscalar $(\omega)$, vector-isovector $(\rho)$ and, in more sophisticated cases (not considered here), also vector-isoscalar $(\delta)$ mesons. For a general review of these types of models, see Ref. [43]. The chosen models fall into two classes: (a) models with constant couplings and nonlinear meson terms, generically called NL models and (b) models with density dependent couplings, generically called DD models. We recall that the nonlinear meson terms have been introduced in order to correctly describe the properties of symmetric saturated nuclear matter, when the coupling constants do not depend on density.

We presently consider the set of models discussed in Ref. [10]. It consists of the NL models: FSU2 [44], FSU2H and FSU2R [45,46], NL3 [47], NL3 $\omega \rho$ [48,49], TM1 [50], TM1 $\omega \rho[48,51,52]$, TM1-2 and TM1-2 $\omega \rho$ [51], and the DD models DD2 [53] and DDME2 [54].

From the astrophysical point of view, the common feature of all these models is the ability to provide NS masses in excess to $2 M_{\odot}$ stars. In regards to the incompressibility parameter of symmetric nuclear matter at saturation, $K_{\infty}$, they span values between $238 \mathrm{MeV}$ (FSU, FSU2R, FSU2H)

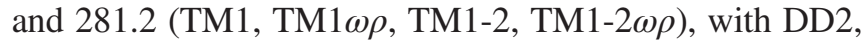
DDME2 and NL3 and NL3 $\omega \rho$ having intermediate values as follows: 242.7, 250.9 and, respectively, $271.5 \mathrm{MeV}$. As is frequent for RMF models, these values are larger than the generally accepted constraints coming from isoscalar giant monopole and dipole resonances in nuclei, $240 \pm 20 \mathrm{MeV}$ [55], or, the more recently, $211.9 \pm 24.5 \mathrm{MeV}$ [56]. In regards to the isovector channel, they fall into two classes: (i) models with moderate values of the slope of the symmetry energy at saturation, which thus comply with a compilation of constraints coming from experimental nuclear physics, $a b$ initio calculations of pure neutron matter and astrophysical observations and are customarily expressed as $40 \lesssim L \lesssim$ $62 \mathrm{MeV}$ [57] or $30 \lesssim L \lesssim 86 \mathrm{MeV}$ [58]. They are FSU2H, FSU2R, NL3 $\omega \rho$, TM1 $\omega \rho$, TM1-2 $\omega \rho$, DD2 and DDME2. These models have $L$ in the range $44-55 \mathrm{MeV}$; (ii) models with high values of the slope of the symmetry energy at saturation, $L \gtrsim 100 \mathrm{MeV}$. They are FSU2, NL3, TM1, TM1-2 with values of $L$ in the range 108-119 MeV. For all these models a table with the corresponding slope values and also other nuclear matter saturation properties can be found in Ref. [10]. At this point we recall that large values of $L$ are still compatible with the Lead Radius Experiment ("PREX") [59], and recent analysis of elliptic flow in heavy ion collisions [60]. The models in the second class are mainly kept for the sake of completeness and because some of the models in the first class have been derived from them by introducing additional meson couplings. It should be pointed out that although most of the models that we consider predict well-accepted nuclear matter properties at saturation density, they are not constrained at high densities, except for the fact that they have to also predict two solar mass stars. The same is true for the hyperonic models which are constrained by properties defined at saturation density. Besides, another source of uncertainty is the density dependence of the hyperonic couplings in the density dependent hyperonic models, which has been considered to behave as the respective nucleonic couplings.

\section{B. Hyperonic EoS with calibrated meson couplings}

The starting point of the meson-exchange RMF model for hypernuclei is the covariant Lagrangian density,

$$
\mathcal{L}=\mathcal{L}_{N}+\mathcal{L}_{Y}+\mathcal{L}_{M},
$$

where $\mathcal{L}_{N}, \mathcal{L}_{Y}$, and $\mathcal{L}_{M}$ respectively stand for the nucleonic, hyperonic and purely mesonic components. In addition to the couplings accounted for when describing infinite nuclear matter-and listed in Sec. II A-, in the case of (hyper)nuclei $\mathcal{L}_{N}$ additionally contains couplings with the photon field $A^{\mu}$. For details, see Ref. [43]. The hyperonic term of Lagrangian density is given by

$$
\mathcal{L}_{Y}=\bar{\Psi}_{Y}\left[\gamma_{\mu} D_{Y}^{\mu}-m_{Y}^{*}+\frac{f_{\omega Y}}{2 m_{Y}} \sigma^{\mu \nu} \partial_{\nu} \omega_{\mu}\right] \Psi_{Y}
$$

with

$$
D_{Y}^{\mu}=i \partial^{\mu}-g_{\omega Y} \omega^{\mu}-g_{\rho Y} \tau_{Y} \cdot \rho^{\mu}-e \frac{\tau_{Y, 3}-1}{2} A^{\mu},
$$

where $m_{Y}^{*}=m_{Y}-g_{\sigma Y} \sigma$ stands for the Dirac effective mass, $e$ is the elementary electric charge, $\tau_{Y}$ is the isospin operator, $\omega^{\mu}$ and $\rho^{\mu}$ are the fields associated to the $\omega$ and $\rho$ mesons and $\Psi_{Y}$ is the $Y$-hyperon field. $g_{\sigma Y}, g_{\omega Y}$ and $g_{\rho Y}$ correspond to the coupling constants of the various meson fields with the $Y$-hyperon. The third term in Eq. (1), proportional to $f_{\omega Y} / 2 M_{Y}$, represents the tensor coupling between the $Y$ hyperon with the $\omega$ field. It impacts the spinorbit splitting and, in principle, can be determined from spectroscopic data. The last term in Eq. (2), proportional to $e$, describes the interaction of the $Y$-hyperon with the Coulomb field and is meaningful for charged hyperons only.

Note that the $\sigma^{*}$ and $\phi$-mesons were not included in Eq. (2) since, for the case of $\Xi$-hyperon of interest here, there are no experimental data that could be exploited in order to fix them. We do, however, include the $\phi$-meson in the next section, devoted to the EoS of stellar matter. In that case the coupling is determined based on $\mathrm{SU}(6)$ flavor symmetry arguments: $g_{\phi \Lambda}=-\sqrt{2} / 3 g_{\omega N}, g_{\phi \Sigma}=-\sqrt{2} / 3 g_{\omega N}$, $g_{\phi \Xi}=-2 \sqrt{2} / 3 g_{\omega N}$ and $g_{\phi N}=0$. This contribution brings repulsion to the interaction and, given that we do 
not include the $\sigma^{*}$-meson, this interaction might be overestimated.

Once the coupling constants are given, the single particle Dirac equations for baryons and the Klein-Gordon equations for mesons and photon are obtained in the mean-field approximation by the variational method [61-63]. The numerical procedure in order to solve the Dirac and KleinGordon variational equations consists in expanding both the meson fields and the baryon single-particle Dirac wave functions in terms of a spherical harmonic oscillator basis. Thus, one has to solve a self-consistent system of nonlinear matrix equations. In the case of the electromagnetic field the Poisson equation is solved directly by using the associated Green's function since the basis expansion method is very slowly convergent. As the translational symmetry is broken in the mean-field approximation, the result has to be corrected for the center of mass motion. The correction is more important for light systems. As in Refs. [8,9], we adopt the microscopic expression $E_{C . M .}=-\left\langle\hat{\mathbf{P}}^{2}\right\rangle / 2 M$, where $M=\sum_{B} M_{B}$ is the total mass of the hypernucleus and $\hat{\mathbf{P}}=\sum_{B} \hat{\mathbf{P}}_{B}$ is the total momentum operator.

The binding energy of the hyperon $Y$ in a nucleus with $A_{n}$ neutrons, $A_{p}$ protons and $A_{Y}$ hyperons is given by the difference between the energies of that hypernucleus and the hypernucleus with $A_{Y}-1$ hyperons. Note that removal of charged hyperons, as $\Xi^{-}$of interest here, implies modification of the number of protons.

Experimental data on hypernuclei binding energy may be used in order to calibrate the interaction between the hyperon and the scalar meson fields [61,64-67]. The issue was most recently addressed by Fortin et al. [8,9], who accounted for a vast collection of experimental data on single- $\Lambda$ hypernuclei in $s$ and $p$ shells and effective nucleonic interactions. These authors have thus confirmed that binding energies are directly related to the well depth of the hyperon at rest in symmetric nuclear matter, as customarily assumed in the literature merely based on heuristic arguments, and that, once the flavor symmetry model is fixed, the value of the hyperon coupling constant represents a fixed fraction of the coupling $g_{\sigma N}$. In the present work we use the $g_{\sigma \Lambda}$ coupling constants obtained in Refs. [8,9].

So far experimental data exist only for two light $\Xi^{-}$hypernuclei: $\Xi^{-} \mathrm{Be}$ and ${ }_{\Xi^{-}} \mathrm{C}$. The former was produced in $\left(K^{-}, K^{+}\right)$reactions on a ${ }^{12} \mathrm{C}$ target [68]. The second, known as the Kiso event [11], corresponds to an intermediate state in the reaction $\Xi^{-}+{ }^{14} \mathrm{~N} \rightarrow{ }_{\Xi^{-}}^{15} \mathrm{C} \rightarrow{ }_{\Lambda}^{10} \mathrm{Be}+{ }_{\Lambda}^{5} \mathrm{He}$. Both sets of data indicate an attractive $N \Xi$ interaction and bound $\Xi$ hypernuclei. See also the recent results in Ref. [69].

Indeed, double differential cross section for ${ }_{\Xi}^{12} \mathrm{Be}$ production was found to be compatible with a $\Xi$-nucleus potential well depth of about $14 \mathrm{MeV}$, within the WoodsSaxon prescription [68]. In regards to the binding energy of $\Xi^{-}$in $\Xi^{-12} \mathrm{Be}$, estimations performed within the cluster model
[70] and the coupled-channels antisymmetrized molecular dynamics approach [71] provide values between 3 and $5.5 \mathrm{MeV}$. As expected, these values depend on the $N \Xi$ interaction. When this interaction is adjusted such as to give a value consistent with the experimental spectrum in Ref. [68], as has been done in Ref. [70], the binding energy is of $\approx 5 \mathrm{MeV}$.

The interpretation of the Kiso event is more problematic, for the final state of the daughter nucleus, ${ }_{\Lambda}^{10} \mathrm{Be}$, was not unambiguously identified. Typically two scenarios are assumed for dealing with data: (a) In the first case it is assumed that ${ }_{\Lambda}^{10} \mathrm{Be}$ is in its ground state. Then, ${ }_{\Xi}^{15} \mathrm{C}$ is considered to be in the state ${ }^{14} \mathrm{~N}($ g.s. $)+\Xi^{-}(1 s)$. (b) In the second scenario ${ }_{\Lambda}^{10} \mathrm{Be}$ is assumed to be produced in an excited state. If this is the case, ${ }_{\Xi}^{15} \mathrm{C}$ corresponds to the state ${ }^{14} \mathrm{~N}($ g.s. $)+\Xi^{-}(1 p)$. The binding energy of $\Xi^{-}$, $B_{\Xi^{-}}=E\left({ }_{\Xi}^{15} \mathrm{C}\right)-E\left({ }^{14} \mathrm{~N}\right)$, has different values in cases (a) and (b). The maximum value corresponds to the case (a) and amounts to $4.38 \pm 0.25 \mathrm{MeV}$. The lower limit, of $1.11 \pm 0.25 \mathrm{MeV}$, corresponds to the second excited state of ${ }_{\Lambda}^{10} \mathrm{Be}$ with an excitation energy of $3.2 \mathrm{MeV}$, as calculated by different models $[72,73]$. Note that the energy spectrum of ${ }_{\Lambda}^{10} \mathrm{Be}$ was recently investigated using $\left(e, e^{\prime} K^{+}\right)$reactions by Gogami et al. [74], who found that the first excited state lies at $\approx 2.7 \mathrm{MeV}$. The two scenarios together with their compatibility with the information obtained for the binding energy of $\Xi$ in ${ }^{12}$ Be have been investigated in Ref. [63], within RMF and Skyrme-Hartree-Fock approaches. The conclusion reached by Sun et al. [63] is that the most plausible interpretation is the one corresponding to ${ }^{14} \mathrm{~N}($ g.s. $)+\Xi^{-}(1 p)$.

In order to constrain the couplings of the $\sigma$ meson to $\Xi$-hyperon we employ the procedure described in Refs. [8,9] and experimental data corresponding to ${ }_{\Xi^{-}}^{15} \mathrm{C}$ [11]. We alternatively assume that ${ }_{\Xi^{-}} \mathrm{C}$ corresponds to the ${ }^{14} \mathrm{~N}$ (g.s. $)+\Xi^{-}(1 s)$ and, respectively, ${ }^{14} \mathrm{~N}$ (g.s. $)+\Xi^{-}(1 p)$ states. In both cases we calculate also $\Xi_{\Xi}^{12} \mathrm{Be}$ and compare with data in Ref. [68].

Other coupling constants are fixed as follows. For the coupling between the $\Xi$ and the $\omega$-meson, we use the SU(6) value $g_{\omega \Xi}=2 / 3 g_{\omega N}$. For the coupling between the $\Xi$ and the $\rho$-meson, we assume $g_{\rho \Xi}=g_{\rho N}$. In DD models, we suppose the same density dependence for hyperon- and nucleon-meson couplings. Let us recall that other calibration constraints have been employed in older works, as proposed in Ref. [75], or more recent works [26]. Finally, in order to get a weaker interaction between $\Xi$ and the nuclear spin orbit, the tensor coupling is included as in Refs. [63,67], with $f_{\omega \Xi}=0.4 g_{\omega N}$.

Since ${ }_{\Xi}^{15} \mathrm{C}$ is constituted by a symmetric nucleus ${ }_{7}^{14} \mathrm{~N}$, with isospin 0 , and a $\Xi$-hyperon, which has nonzero isospin, the $\rho$-meson field is finite due to the selfinteraction of the $\Xi$ with itself. To remove this spurious contribution, we follow the procedure proposed in 
TABLE I. Coupling constant fraction $x_{s \Xi}=g_{\sigma \Xi} / g_{\sigma N}$, well depth of $\Xi^{-}$at rest in symmetric matter at saturation density $\left(U_{\Xi}^{(N)}\right)$ and, respectively, binding energy of $\Xi$ in ${ }_{\Xi 1 s}^{12} \mathrm{Be}$, as obtained from the fit of the binding energy of ${ }_{\Xi}^{15} \mathrm{C}$. Results corresponding to the hypothesis according to which ${ }_{\Lambda}^{10} \mathrm{Be}$ is produced in the ground state or, alternatively, the first excited state are reported in columns $2-4$ and, respectively, 5-7. The binding energies of ${ }_{\Xi 1 s}^{15} \mathrm{C}$ and ${ }_{\Xi 1 p}^{15} \mathrm{C}$ are $\mathrm{BE}_{\Xi}=4.4 \mathrm{MeV}$ and $\mathrm{BE}_{\Xi}=1.1 \mathrm{MeV}$. For the second hypothesis, also the energy of ${ }_{\Xi 1 s}^{15} \mathrm{C}$ is provided, on the last column. Results correspond to different nucleon effective interactions.

\begin{tabular}{|c|c|c|c|c|c|c|c|}
\hline \multirow[b]{2}{*}{ Model (MeV) } & \multicolumn{3}{|c|}{${ }_{\Xi 1 s}^{15} \mathrm{C}(\mathrm{BE}=4.4 \mathrm{MeV})$} & \multicolumn{4}{|c|}{${ }_{\Xi 1 p}^{15} \mathrm{C}(\mathrm{BE}=1.1 \mathrm{MeV})$} \\
\hline & $x_{s \Xi}$ & $U_{\Xi}^{(N)}$ & $1_{\Xi 1 s} \mathrm{Be}(\mathrm{MeV})$ & $x_{s \Xi}$ & $U_{\Xi}^{(N)}(\mathrm{MeV})$ & ${ }_{\Xi 1 s}^{12} \mathrm{Be}(\mathrm{MeV})$ & ${ }_{\Xi 1 s}^{15} \mathrm{C}(\mathrm{MeV})$ \\
\hline $\mathrm{DD} 2$ & 0.304 & -11.10 & 2.35 & 0.320 & -17.50 & 6.48 & 9.02 \\
\hline DDME2 & 0.306 & -12.49 & 2.38 & 0.321 & -18.78 & 6.31 & 8.83 \\
\hline FSU2R & 0.296 & -11.80 & 2.51 & 0.316 & -17.51 & 5.87 & 8.12 \\
\hline FSU2 & 0.296 & -10.00 & 2.64 & 0.311 & -15.69 & 6.15 & 8.19 \\
\hline FSU2H & 0.296 & -10.00 & 2.68 & 0.310 & -15.47 & 6.47 & 7.91 \\
\hline TM1 & 0.295 & -9.78 & 2.59 & 0.310 & -14.93 & 5.48 & 7.69 \\
\hline $\mathrm{TM} 1 \omega \rho$ & 0.295 & -9.80 & 2.51 & 0.310 & -14.94 & 5.34 & 7.68 \\
\hline TM1-2 & 0.292 & -8.71 & 2.58 & 0.309 & -14.62 & 6.62 & 8.79 \\
\hline 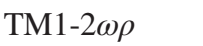 & 0.292 & -8.74 & 2.59 & 0.309 & -14.63 & 6.56 & 8.77 \\
\hline NL3 & 0.296 & -9.88 & 2.84 & 0.310 & -15.36 & 7.31 & 7.93 \\
\hline NL3 $\omega \rho$ & 0.296 & -9.90 & 2.73 & 0.311 & -15.39 & 7.17 & 7.93 \\
\hline
\end{tabular}

Refs. $[63,67]$. It consists in performing two calculations of the hypernucleus energy. (1) In the first one, the coupling of the $\rho$-meson to the nucleons is put to 0 while the coupling of the $\rho$-meson to the $\Xi$-hyperon is kept fixed. The corresponding energy is $E\left(g_{\rho N}=0, g_{\rho \Xi}\right)$. (2) In the second case, the coupling constants of the $\rho$-meson to both nucleons and $\Xi$-hyperon are put to 0 . The corresponding energy is $E\left(g_{\rho N}=0, g_{\rho \Xi}=0\right)$. The spurious energy is given by $E_{s p}=$ $E\left(g_{\rho N}=0, g_{\rho \Xi}\right)-E\left(g_{\rho N}=0, g_{\rho \Xi}=0\right)$ and can be straightforwardly removed from the full calculation. We employ this procedure to correct the energies of both ${ }_{\Xi}^{15} \mathrm{C}$ and ${ }_{\Xi}^{12} \mathrm{Be}$.

The values of the coupling constant $g_{\sigma \Xi}$, expressed as the fraction $x_{s \Xi}=g_{\sigma \Xi} / g_{\sigma N}$, obtained from the fit of the binding energy of the $\Xi$-hyperon in the ${ }_{\Xi}^{15} \mathrm{C}$ hypernucleus are given in Table I. Different effective nucleon interactions, introduced in Sec. II A, are considered. The two scenarios which assume that $\Xi^{-}$occupies the $1 s$ or, alternatively, the $1 p$ state of ${ }^{14} \mathrm{~N}$ are considered separately. They correspond to the binding energies $\mathrm{BE}=4.4 \mathrm{MeV}$ and, respectively, $\mathrm{BE}=1.1 \mathrm{MeV}$. Also given are the values of $U_{\Xi}^{(N)}$, the well depth of $\Xi$ at rest in symmetric saturated nuclear matter, and the binding energy of the $\Xi$ hyperon in the hypernucleus ${ }_{\Xi}^{12} \mathrm{Be}$. Finally the last column lists, for comparison, the binding energy of $\Xi$ in the $1 s$ state of $\Xi^{-15} \mathrm{C}$ obtained when the coupling constant given in the fifth column is used. If we take into account that the experimental data of ${ }_{\Xi}^{12} \mathrm{Be}$ [68] have been interpreted as compatible with $U_{\Xi}\left(n_{0}\right) \sim-14 \mathrm{MeV}$, our results confirm the conclusion of Ref. [63], suggesting as the most plausible scenario the one in which ${ }_{\Xi}^{15} \mathrm{C}$ is produced in an excited state. In regards to the binding energy of $\Xi$ in ${ }_{\Xi}^{12} \mathrm{Be}$, the situation is less clear as the values provided by Refs. [70,71], in the range $3-5.5 \mathrm{MeV}$, sit in between the values we obtained for the two scenarios. We nevertheless note that three interactions (FSU2R, TM1 and TM1 $\omega \rho$ ) provide, for the second scenario, values similar to those of Refs. [70,71].

The binding energy of the $\Xi$-hyperon in the hypernucleus ${ }_{\Xi}^{15} \mathrm{C}$ is plotted in Fig. 1 as a function of the $U_{\Xi}^{(N)}$ potential (top panels) and, respectively, $x_{s \Xi}$ (bottom panels). Left (right) panels correspond to the assumptions according to which $\Xi$ occupies a $1 s$ (1p) state. The different nucleonic effective interactions presented in Sec. II A are considered. A gray band identifies the binding energy obtained in the Kiso event [11]. Some conclusions are in order: (a) if ${ }_{\Xi}^{15} \mathrm{C}$ is in the ground state, i.e., $\Xi$ occupies the $1 s$ state, $-12.5 \lesssim U_{\Xi}^{(N)} \lesssim-8.7 \mathrm{MeV}$, with the most attractive values corresponding to the two DD models and FSU2R; this corresponds to $0.295 \leq x_{s \Xi} \leq 0.306$; see Table I; (b) if ${ }_{\Xi}^{15} \mathrm{C}$ is in an excited state, i.e., $\Xi$ occupies the $1 p$ state, $U_{\Xi}^{(N)}$ is more attractive, $-18.8 \lesssim U_{\Xi}^{(N)} \lesssim-14.6 \mathrm{MeV}$; again, the most attractive values correspond to the two DD models and FSU2R; the coupling constants have larger values, $0.31 \leq x_{s \Xi} \leq 0.32$. The relative stability of $x_{s \Xi}$ to the modification of the nucleonic EoS, for each considered scenario, reflects the relatively small dispersion among the considered EoS, over the subsaturation density domain explored by a hyperon bound in a nucleus. Note that a similar situation corresponds, according to [8-10], also to $x_{s \Lambda}$ and the explication is the same.

Contrary to what occurs for $\Lambda$ and $\Xi$, there is no hypernuclear data on which the $\Sigma \mathrm{N}$ interaction can be tuned. As a consequence we treat $g_{\sigma \Sigma}$ as a free parameter and vary its values such as to explore $-10 \leq U_{\Sigma}^{(N)} \leq 40 \mathrm{MeV}$. 

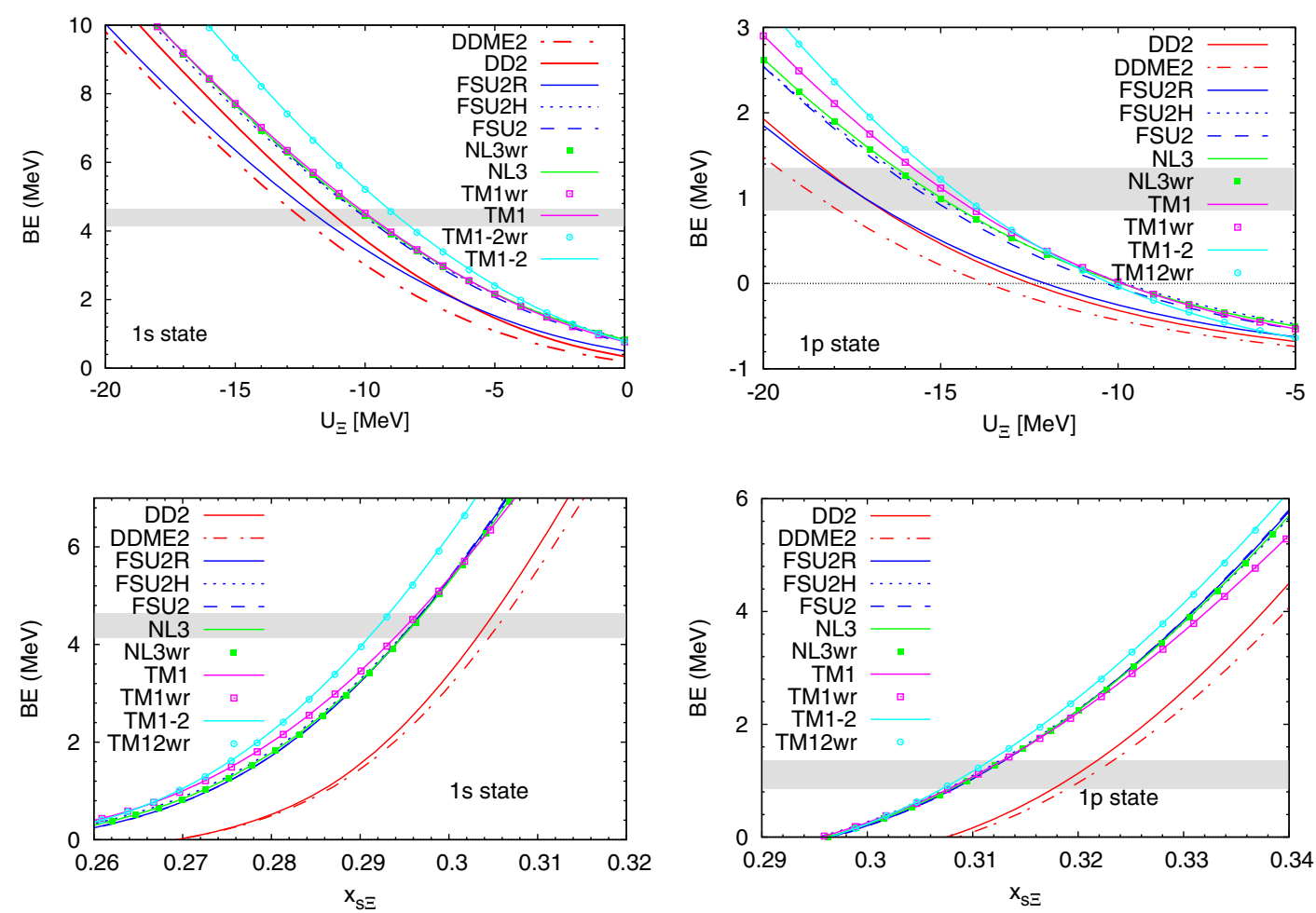

FIG. 1. Binding energy of ${ }_{\Xi^{-}}^{15} \mathrm{C}$ as a function of $U_{\Xi}^{(N)}$ (top panels) and $x_{s \Xi}$ (bottom panels), under the assumptions that $\Xi^{-}$occupies a $1 s$ state (left panels) or, alternatively, a $1 p$ state (left panels). Note that the scales are not the same in the different panels. Gray band: experimental binding energy of the Kiso event.

We recall that, according to Ref. [13], $U_{\Sigma}^{(N)}\left(n_{0}\right) \approx$ $30 \pm 20 \mathrm{MeV}$. As assumed for the couplings between $\Xi$ and $\omega$ and $\rho$ mesons and for the same reasons, $g_{\omega \Sigma}=$ $2 / 3 g_{\omega N}$ and $g_{\rho \Sigma}=g_{\rho N}$.

\section{PROPERTIES OF HYPERNUCLEAR COMPACT STARS}

In the following we discuss the properties of hypernuclear compact stars built upon the calibrated EoS discussed in Sec. II. In particular, we analyze the effect of the different nucleon effective interactions and $U_{\Sigma}^{(N)}$-potential values on the onset and abundances of hyperons as well as on the maximum mass, radii, tidal deformability and moment of inertia.

In the following we use unified EoS for neutron star matter for the 11 parametrizations in this work. We employ crust models computed consistently with the core [10,27] following the approach presented in the first reference. EoS tables are available as Supplemental Material [76] to this paper assuming that the $\Xi^{-}$hyperon in ${ }_{\Xi}^{15} \mathrm{C}$ of the Kiso event is in a $1 p$ state, for each of the $11 \mathrm{RMF}$ models and four values of the $U_{\Sigma}$ potential: $-10,10,30$ and $50 \mathrm{MeV}$.

For a spherical star in hydrostatic equilibrium we determine the mass-radius relation by solving the TolmanOppenheimer-Volkoff [77,78] equations. The radius of some NSs has been determined, most recently by two teams who modeled the pulsed x-ray emission of the millisecond pulsar PSR J0030 + 0451 [79,80]. However uncertainties in the derived constraints and in the modeling of the source itself are still large and so far no strong constraint on the radius of NS has been obtained (see e.g., $[81,82])$. However the determination of the radius of a few NSs with a precision of a few percent is expected from the currently operating NICER mission [83] and also from future X-ray observatories like Athena X-ray telescope [84] and eXTP [85].

The moments of inertia $I$ and tidal deformabilities $\Lambda$ are calculated as following Refs. [86,87] and Ref. [88], respectively. Some constraints on the deformabilities of the two NS that composed the binary before they merge during the GW170817 event have been obtained thanks to the multimessenger observations (see Refs. [89,90] for the latest results), and many more are expected in the near future from the current and future observational runs of the LVC collaborations. As far as the moment of inertia is concerned, no measurement has been obtained so far. However it could be measured in a binary of two radio pulsars, such as PSR J0737-3039. It could not be achieved for this system so far as the radio beam of one of the NSs cannot be observed anymore due to precession. With more observations with current radiotelescopes and future ones like the SKA [91], the number of known pulsars is expected to increase by orders of magnitude, including many thousands 
of millisecond pulsars, and among them possibly binary systems with two pulsars.

Figure 2 gives a general summary of the properties of the NSs built upon the considered models. Regarding observational constraints on the maximum mass we note that, thanks to radio observations of the Shapiro delay, the mass of MSP $\mathrm{J} 0740+6620$ has been determined to be $2.14_{-0.18}^{+0.20} M_{\odot}$ at a 2- $\sigma$ level (equivalently $95.4 \%$ credibility interval), i.e., $1.96<M / M_{\odot}<2.34$ [30]. We consider that the uncertainty in this measurement is still too large to use this mass as a strong constraint. Let us recall indeed that the mass of PSR J1614 - 2230 inferred from Shapiro delay was initially determined to be $1.97 \pm 0.04 M_{\odot}(1-\sigma$ level) [18]. After more data were accumulated this number went down to $M=$ $1.908 \pm 0.016 M_{\odot}$ (also $1-\sigma$ level), which is compatible with the previous reported mass at more than 1 standard deviation. Consequently in this work we only consider and plot the mass constraints from PSR J1614 - 2230 and J0348 + 0432. We note that among all the models considered in this paper, DD2, DDME2, FSU2H, NL3 and NL3 $\omega \rho$ are compatible with the mass constraint from these objects. We also show in the $M-R$ plot the mass and radius constraints at the $1-\sigma$ level obtained for PSR J0030 + 0451 by two teams analyzing NICER data $[79,80]$. The stiffest EoS NL3, which is already ruled out because of its too large slope of the symmetry energy at saturation, is not compatible with these measurements, while TM1 and TM1-2 are marginally consistent.

In the middle panel of Fig. 2 we plot for indication the limits imposed on $\Lambda_{1.4}$ taken from Ref. [89]. These have been deduced from the effective $\tilde{\Lambda}$ obtained within the waveform model PhenomPNRT at a $90 \%$ confidence level. None of the models we use satisfy this constraint. However, in Ref. [90] the authors show the dependence of the analysis of the GW170817 observation on the waveform model, and, in particular, the TaylorF2 model predicts effective tidal deformabilities larger by $\sim 100$. New observations are needed to impose stricter constraints.

Figure 3 represents strangeness fractions $N_{S} / N_{B}$ as a function of the star mass. Baryonic and strangeness numbers entering the definition of the strangeness fraction are defined as

$$
\begin{aligned}
& N_{B}=4 \pi \int d r \frac{n_{i} r^{2}}{\sqrt{1-2 m(r) / r}}, \\
& N_{S}=\frac{4 \pi}{3} \int d r \frac{q_{S i} n_{i} r^{2}}{\sqrt{1-2 m(r) / r}},
\end{aligned}
$$

where $n_{i}$ and $q_{S i}$ stand for particle number density and, respectively, strangeness charge of particle $i$, and $m(r)$ denotes the gravitational mass corresponding to the radial coordinate $r$. The dependence of NS properties and strangeness composition on the magnitude of the $\Sigma$-N interaction potential is illustrated by using different colors and thicknesses, the thinner the line the more repulsive the potential.
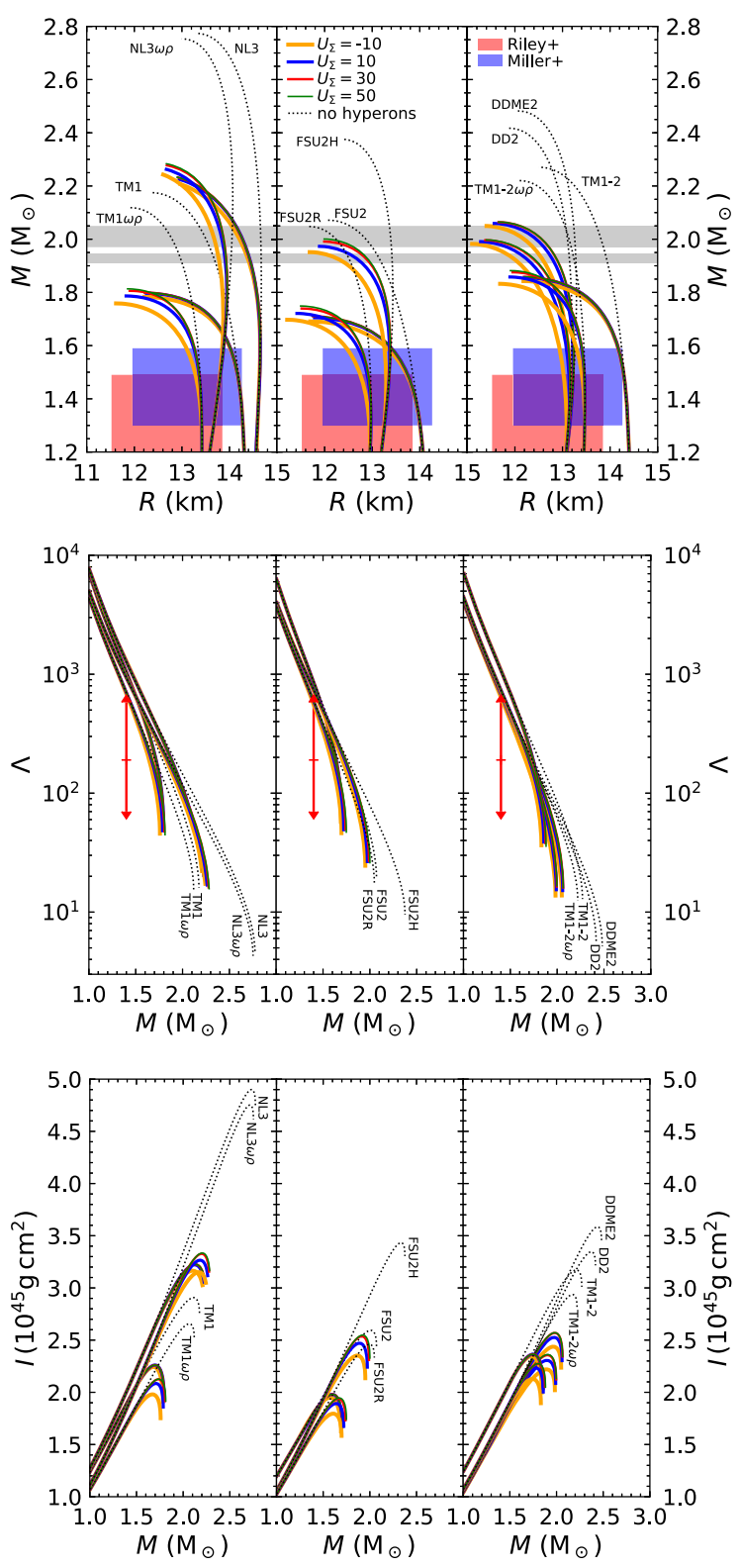

FIG. 2. Mass-radius diagrams (top panels) and tidal deformabilities (second middle panels) and moments of inertia (third bottom panels) as a function of the star mass expressed in units of solar masses, for hypernuclear stars with calibrated $\Lambda$ and $\Xi$ interactions. The different nucleonic effective interactions discussed in Sec. II A are considered. Predictions corresponding to different values of $U_{\Sigma}^{(N)}$ are plotted with different colors: -10 (gold), 10 (blue), 30 (red) and $50 \mathrm{MeV}$ (green) and line thicknesses: the thiner the line the more repulsive the potential. Predictions corresponding to purely nucleonic stars are illustrated by dotted curves. The horizontal bands on $M-R$ diagrams correspond to the mass measurements of PSR $\mathrm{J} 1614-2230$ and $\mathrm{J} 0348+0432$ with a $1-\sigma$ uncertainty. The colored rectangles correspond to the mass and radius constraints at the $1-\sigma$ level obtained for PSR $\mathrm{J} 0030+0451$ by two teams analyzing NICER x-ray data $[79,80]$. For indication, the red vertical bars on $\Lambda-M$ correspond to limits obtained on the tidal deformability of a $1.4 M_{\odot} \mathrm{NS}, 70<\Lambda_{1.4}<580$, as derived from the observation of GW170817 by the LVC collaboration [89], using the waveform model PhenomPNRT. 


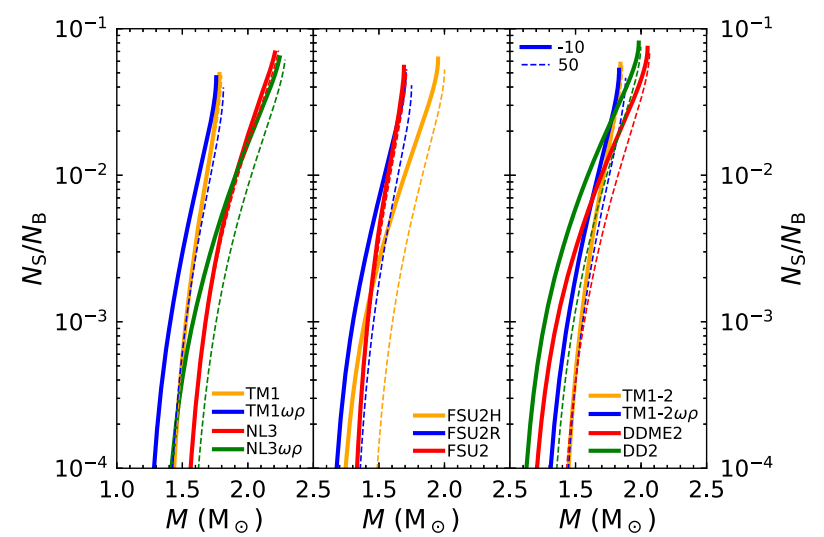

FIG. 3. Strangeness fraction as a function of the star mass expressed in units of solar masses for the same models considered in Fig. 2. The thick lines correspond to $U_{\Sigma}^{(N)}=-10 \mathrm{MeV}$ and the thin ones to $+50 \mathrm{MeV}$.

It comes out that the most important role is played by the nucleonic sector. The reason is that nucleons represent the dominant component. Quite remarkably, also the modifications brought by nucleation of $\Sigma$-hyperons and the associated value of $U_{\Sigma}^{(N)}$ show strong dependence on the nucleonic EoS. For instance, models with large values of the slope of the symmetry energy (e.g., TM1, TM1-2, NL3 and FSU2) show very little sensitivity of geometric, deformability and chemical composition to the value of $U_{\Sigma}^{(N)}$. At variance with them, models with moderate $L$ values lead to smaller NS radii and masses, when attractive or less repulsive $\Sigma \mathrm{N}$ potentials are assumed. From Fig. 3 one may see that the maximum strangeness fraction reached in NS cores does not depend on $U_{\Sigma}^{(N)}$. The strangeness related quantity which does depend on $U_{\Sigma}^{(N)}$ is the density and, implicitly, the NS mass where strangeness sets in. As is easy to anticipate, attractive or less repulsive potentials favor early nucleation of $\Sigma$.

In Table II, we have compiled, for each model and each value of the $U_{\Sigma}^{(N)}$ potential the information covering several properties of neutron stars: maximum mass and respective central baryonic number density, onset density of the three hyperonic species and threshold densities of nucleonic and various hyperonic dUrca channels. Also given are the NS masses with central densities equal to these values.

In the following, we discuss these results.

(i) Some of the considered models, e.g., FSU2R, FSU2, TM1, TM1 $\omega \rho$, TM1-2 and TM1-2 $\omega \rho$, are not able to reach the $2 M_{\odot}$ lower bound of maximum NS mass, when hyperons are introduced.

(ii) Irrespective of the nucleonic EoS, the only hyperonic species that are present are $\Lambda, \Sigma^{-}$and $\Xi^{-}$. The explanation relies on the attractive character of $\Lambda \mathrm{N}$ and $\Xi N$ interactions and dominance of negatively charged particles. Note that other models of hypernuclear compact stars allow also for $\Xi^{0}$ [27]. (iii) For most of the NL models studied here only two species of hyperons nucleate in the core, the $\Lambda$ and $\Sigma^{-}$hyperons. However for repulsive enough $U_{\Sigma}^{(N)}$ potential the $\Xi^{-}$nucleates either instead of the $\Sigma^{-}$for the TM1, TM1-2 and NL3 models or in addition to it. For DD models the three hyperons $\Lambda, \Sigma^{-}$and $\Xi^{-}$ appear, the latter species the last except for all models but one.

(iv) For models with a large $L$, e.g., TM1, TM1-2, NL3 and FSU2, the nucleonic dUrca is insensitive to the onset of hyperons. The reason is that it becomes active well before the onset of any hyperon species. We recall that the nucleonic dUrca process corresponds to the neutron $\beta$-decay followed by the electron capture on the proton: $n \rightarrow p+e^{-}+\bar{\nu}_{e}$ and $p+e^{-} \rightarrow n+\nu_{e}$, which operates when the Fermi momenta of involved baryons and charged lepton verify the triangle inequality: $p_{F, i}+p_{F, j} \geq$ $p_{F, k}$ [92]. This latter condition also applies to the hyperonic dUrca processes [93].

(v) With the exception of the above cited models, attractive $U_{\Sigma}^{(N)}$ potentials modify NS composition such that the nucleonic dUrca threshold is shifted to lower densities.

(vi) NL and DD models provide different results in regard to the nucleonic dUrca process. More precisely, DD models either completely forbid this process or allow for it at densities beyond the central density of the maximum mass, which is equivalent with saying that it never operates. All NL models allow for nucleonic dUrca. Depending on the value of the symmetry energy it starts to operate at densities slightly above $n_{0}$ or several times $n_{0}$.

(vii) DD models only allow for hyperonic dUrca [93]. The allowed processes are $\Lambda \rightarrow p+e+\tilde{\nu}_{e}, \Sigma^{-} \rightarrow \Lambda+$ $e+\tilde{\nu}_{e}$ and, for repulsive values of $U_{\Sigma}^{(N)}$, also $\Xi^{-} \rightarrow$ $\Lambda+e+\tilde{\nu}_{e}$ (not mentioned in Table II) For strongly repulsive $U_{\Sigma}^{(N)}, \Lambda \rightarrow p+e+\tilde{\nu}_{e}$ sets in much before $\Sigma^{-} \rightarrow \Lambda+e+\tilde{\nu}_{e}$. For less repulsive $U_{\Sigma}^{(N)}$ the two processes have close density thresholds and, thus, compete. For $U_{\Sigma}^{(N)} \geq 20 \mathrm{MeV}, \Xi^{-} \rightarrow \Lambda+e+\tilde{\nu}_{e}$ sets in at densities of the order of $0.37-0.46 \mathrm{fm}^{-3}$, which corresponds to $1.46 M_{\odot} \leq M \leq 1.66 M_{\odot}$.

(viii) In NL models with $L \sim 50-60 \mathrm{MeV}$ the hyperonic dUrca starts operating before the nucleonic dUrca. Repulsive $U_{\Sigma}^{(N)}$ values shift $\Lambda \rightarrow p+e+$ $\tilde{\nu}_{e}$ to lower densities.

(ix) $U_{\Sigma}$ defines the process that first operates: a less repulsive potential favors $\Sigma^{-} \rightarrow \Lambda+e+\tilde{\nu}_{e}$, which is 20 times more efficient than $\Lambda \rightarrow p+e+\tilde{\nu}_{e}$ [93].

(x) With the exception of NL3 $\omega \rho$, under the assumption that $U_{\Sigma}^{(N)}$ is repulsive, the $\Lambda \rightarrow p+e+\tilde{\nu}_{e}$ process starts operating at $n \approx 2 n_{0}$, which corresponds to 


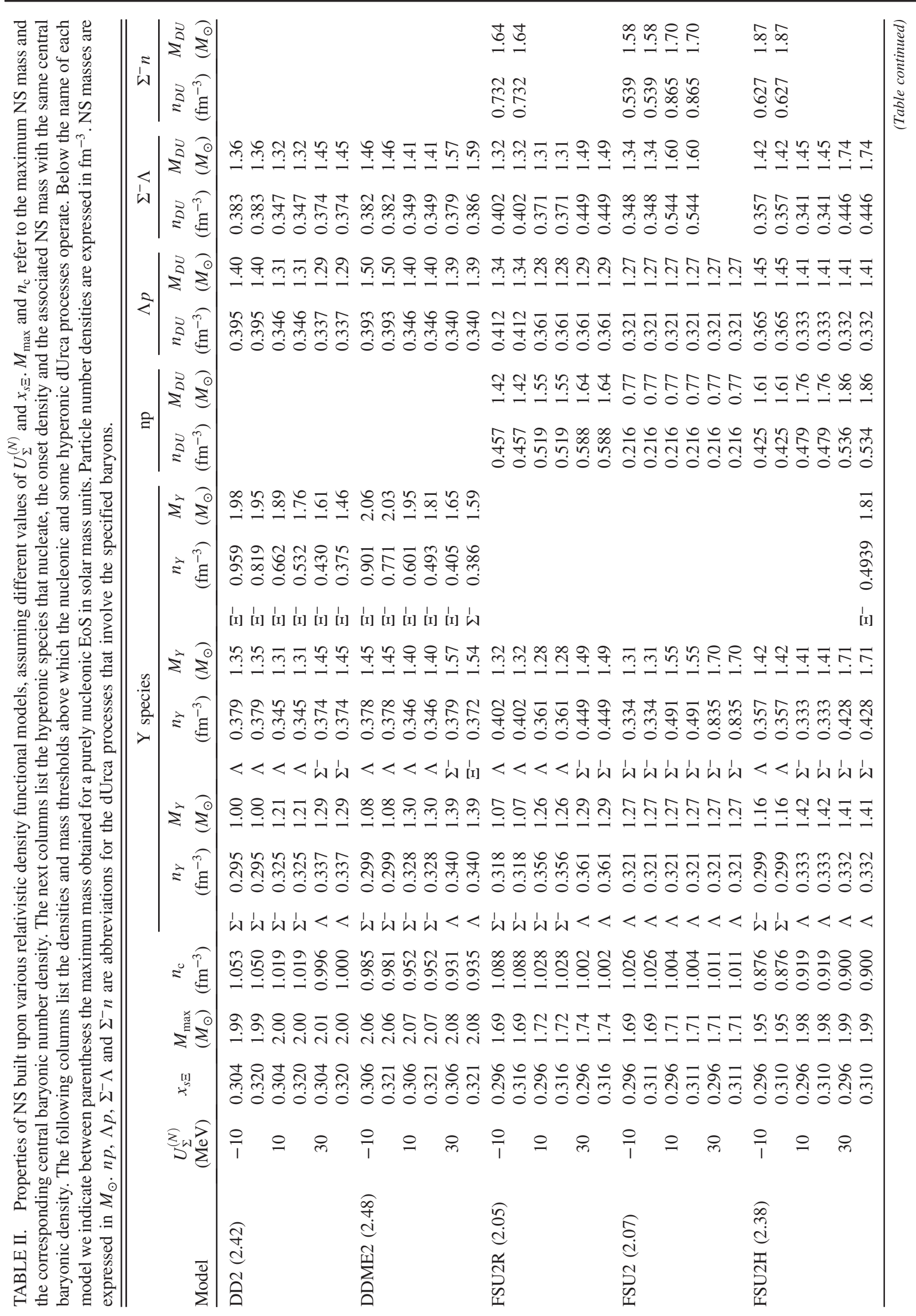




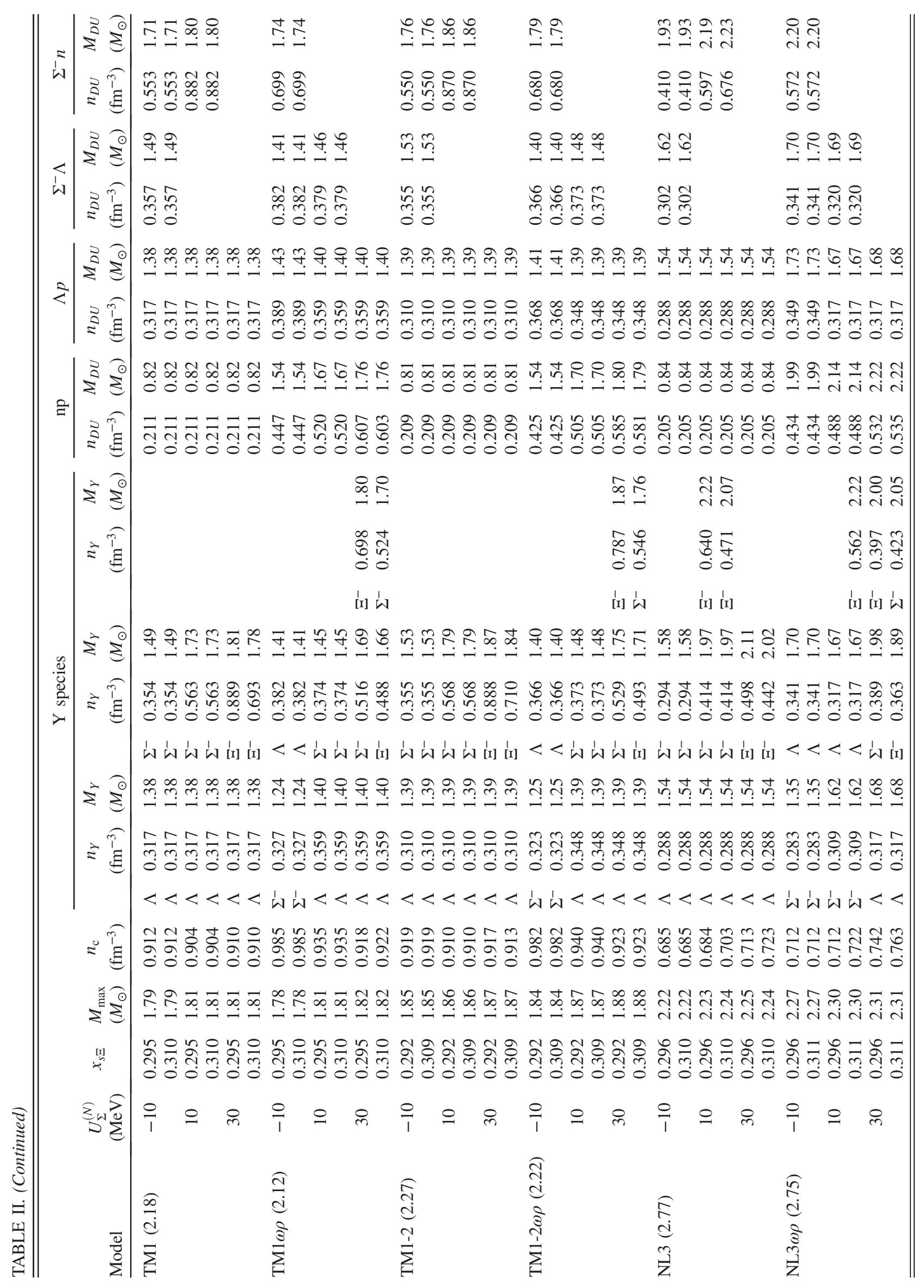



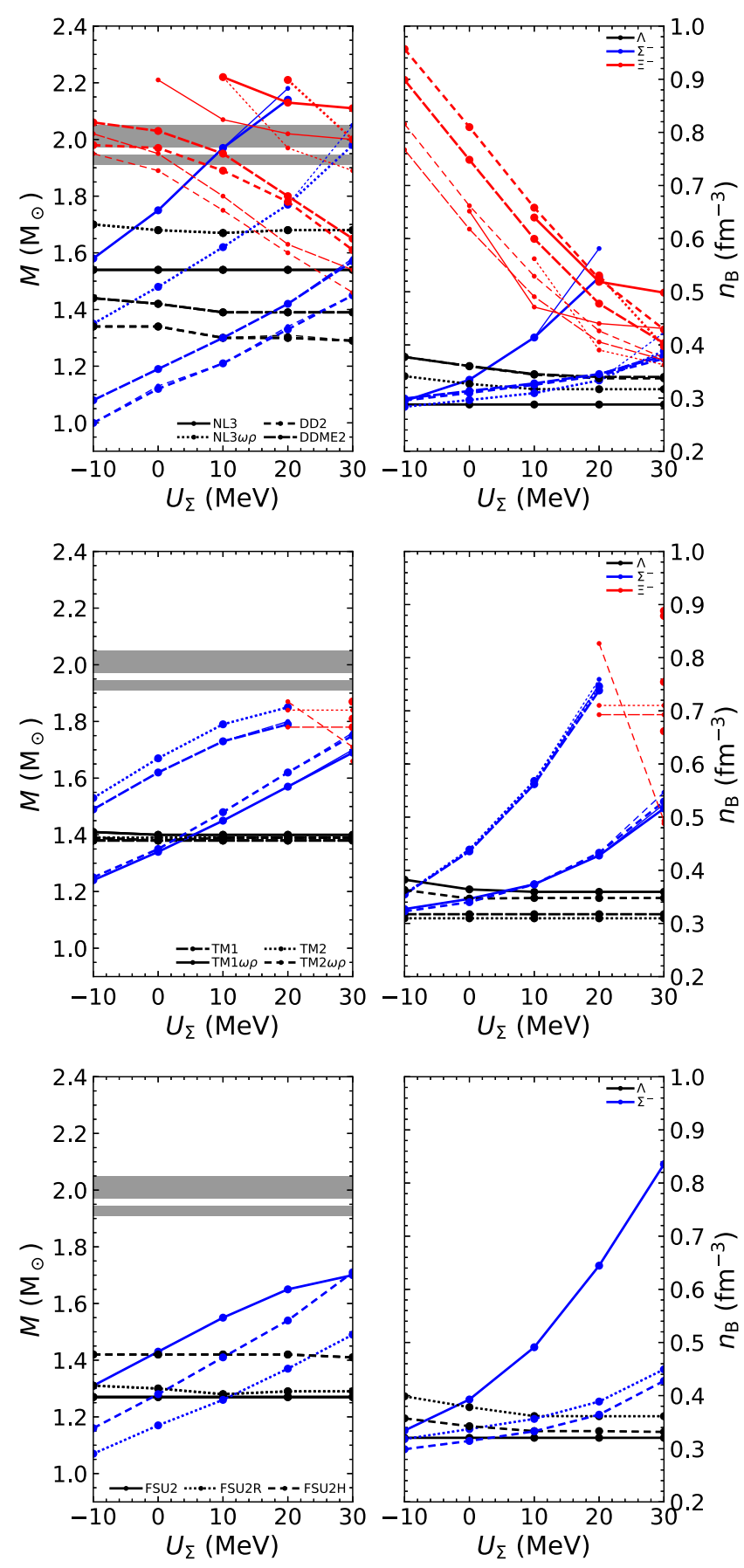

FIG. 4. $U_{\Sigma}^{(N)}$ dependence of the onset density (right panel) and corresponding NS mass with this central density (left panel) of the hyperonic species that nucleate in NS cores. The same models as in Fig. 1 are considered. The horizontal gray strips on the left panel correspond to the mass measurements of PSR J1614 2230 and $\mathrm{J} 0348+0432$ with a $1-\sigma$ uncertainty.

$M / M_{\odot} \approx 1.3-1.4 M_{\odot}$. The relative stability of this threshold is attributable to the constraints imposed to nuclear matter around the $n_{0}$ and $\Lambda N$ potential.

Figures 4 and 5 illustrate the dependence of some quantities reported in Table II on the nucleon effective interaction and $U_{\Sigma}^{(N)}$ potential. These are the densities at
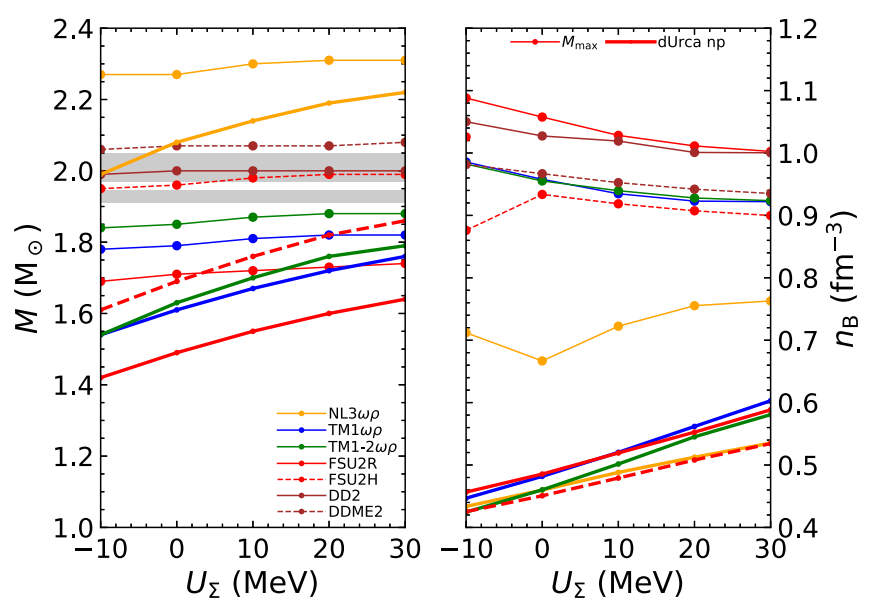

FIG. 5. Left: dependence on $U_{\Sigma}^{(N)}$ of the NS maximum mass $M_{\max }$ (thin lines) and of the minimum NS mass which allows nucleonic dUrca to operate (thick lines). The horizontal gray strips correspond to the mass measurements of PSR J16142230 and J0348 +0432 with a 1- $\sigma$ uncertainty. Right: dependence on $U_{\Sigma}^{(N)}$ of the central density at the maximum mass (thin dashed lines) and of the onset density of nucleonic dUrca (thick solid lines).

which the three hyperonic species nucleate in NS matter and the corresponding NS masses (Fig. 4) and, respectively, the density and corresponding NS masses where nucleonic dUrca becomes active (Fig. 5). Figure 4 shows that the onset density of $\Lambda$ s depends little on $U_{\Sigma}^{(N)}$ and the nucleonic EoS. The explanation of the first feature is that, with the exception of attractive $U_{\Sigma}^{(N)}$ values and FSU2H, $\Lambda$ onset before $\Sigma$. The explanation of the second one is that, up to $n_{\Lambda}$, the nucleonic EoS is relatively well constrained. Despite small dispersion on $n_{\Lambda}$, the mimimum NS mass that accommodates $\Lambda \mathrm{s}$ varies over $0.4 M_{\odot}$. The size of this interval reflects the integrated variation among the EoS, especially in the isovector channel, up to $n_{\Lambda}$. Nucleation of $\Sigma$ depends much on both $U_{\Sigma}^{(N)}$ and nucleon-nucleon effective interactions. In terms of density the domain of variation is $\approx 0.5 \mathrm{fm}^{-3}$ wide, while in terms of NS masses it is $\approx 1.2 M_{\odot}$. Nucleation of $\Xi$, which appear only in some models, is inversely correlated with that of $\Sigma^{-}$. Even larger uncertainties affect these latter quantities and the explanation is obviously due to the increased uncertainties that affect the EoSs as the density increases. Figure 5 illustrates the already discussed huge dispersion that concerns the onset density and mass of the nucleonic dUrca process. The variation of this quantity with the nucleon-nucleon effective interaction stems from the isovector channel. The variation with $U_{\Sigma}^{(N)}$ reflects the way in which negatively charged particles affect the whole composition of matter and, implicitly, the relative abundances of neutrons, protons and electrons. 


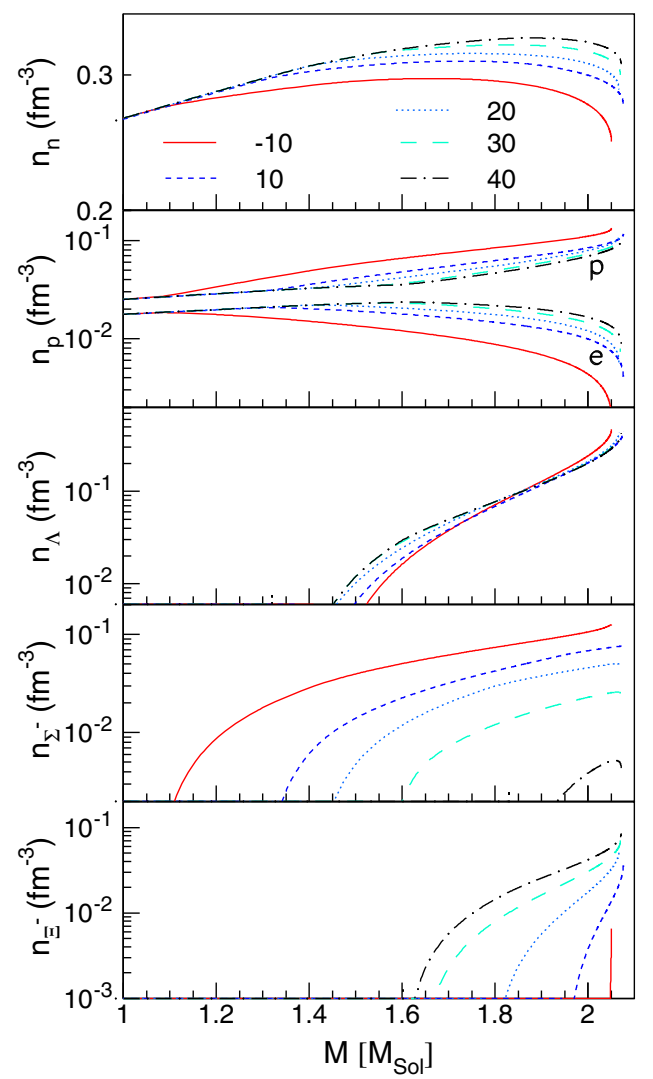

FIG. 6. For the DDME2 model, from top to bottom, partial densities of neutrons, protons, $\Lambda \mathrm{s}, \Sigma \mathrm{s}$ and $\Xi \mathrm{s}$ in the center of the star as a function of the gravitational mass in units of solar masses. The different curves correspond to different values of the $U_{\Sigma}^{(N)}$, as mentioned in the key legend (in $\mathrm{MeV}$ ). Also plotted in the second panel are the electron densities.

Modification of chemical composition is expected to impact the thermal evolution of isolated and accreting neutron stars. The extent to which one may constrain the effective interactions from effective surface temperature will be considered in a future work.

With the aim of understanding how $U_{\Sigma}^{(N)}$ affects NS chemical composition and, consequently, the dUrca threshold Fig. 6 illustrates, for the DDME2 parametrization, the individual central particle number densities $n_{\mathrm{i}}$ as a function of the gravitational mass (in units of solar masses) for different values of $U_{\Sigma}^{(N)}$ between -10 and $40 \mathrm{MeV}$. The considered species are neutrons, protons, electrons, $\Lambda \mathrm{s}, \Sigma \mathrm{s}$ and $\Xi$ s. Attractive or less repulsive values of $U_{\Sigma}^{(N)}$ favor the onset of $\Sigma^{-}$at lower values of $n_{B}$. By partially replacing the electrons, which compensate the positive electric charge of protons, $\Sigma^{-}$s modify both neutron and proton densities, as the first two panels confirm. Smaller values of $n_{n}$ together with larger values of $n_{p}$ act in the direction of allowing the nucleonic dUrca to operate at lower densities. Chemical equilibrium with unconserved strangeness asks that $\Lambda$ chemical potential is equal with the neutron one. As a result, $n_{\Lambda}$ qualitatively follows the evolution of $n_{n}$.
Quantitatively, the less abundant $\Lambda$ s are less affected than the more abundant neutrons. Based on similar arguments one could expect that $n_{\Xi^{-}}$follows $n_{\Sigma^{-}}$. The bottom panel of Fig. 6 shows the opposite; i.e., the more attractive $U_{\Sigma}^{(N)}$ is the smaller the amount of $\Xi$-hyperons. The effect is attributable to the net neutrality condition, where the role of $\Xi$ s is overtaken by $\Sigma$ s.

The most important consequence of hyperon nucleation in the NS core is the drastic reduction of the maximum mass. Other quantities, like NS radius, tidal deformability and moment of inertia are also affected though to a much lesser extent, as illustrated in Fig. 2. The question raised is which is the relation between the strangeness density or fraction and the magnitude by which different parameters that characterize hypernuclear stars deviate from those that characterize purely nucleonic stars. Figure 7 shows, in log$\log$ scale, the relative deviations which affect the radii (top panels), the tidal deformabilities (middle panels) and the moments of inertia (bottom panels) for NSs with masses equal to $1.6 M_{\odot}, 1.7 M_{\odot}$ and $1.8 M_{\odot}$. The predictions corresponding to different nucleonic models are plotted with different colors, while different symbols signal the different values of $U_{\Sigma}^{(N)}=-10,10,30$ and $50 \mathrm{MeV}$. It comes out that each of the three quantities is strongly correlated with $N_{S} / N_{B}$. The Pearson correlation factor $r$, indicated on each panel, is only slightly smaller than one indicating the strong correlations between the quantities of interest in each panel. Moreover, the relation between $\log \left(\left|F_{\text {nucl }}-F_{\text {hyp }}\right| / F_{\text {nucl }}\right)$ and $\log \left(N_{S} / N_{B}\right)$, where $F=R$, $\Lambda, I$, is linear. The parameters of the linear fit are mentioned on each panel. In all cases, the slopes of the lines are slightly larger than 1 .

These correlations can be understood by considering that all the above quantities $F=R, \Lambda, I$ depend on $R$ to a given power $n,(n=1,5$ and 2 for $R, \Lambda$ and $I)$. Assuming that $R$ gets modified by $\delta R, \delta F \sim n R_{\text {nucl }}^{(n-1)} \delta R$. In its turn, $\delta R$ depends linearly on $\delta M$,

$$
\delta R=-\left(\frac{\partial R}{\partial M}\right)_{M_{\text {nucl }}} \delta M,
$$

where $\delta M$ stands for the reduction of NS mass due to the onset of hyperons, $\delta M=M_{\text {nucl }}-M_{\text {hyp }}$. Finally, for small strangeness fractions, $\delta M \sim N_{S} / N_{B}$.

According to Fig. 7, the modifications entailed by hyperons on the radius, tidal deformability and moment of inertia of NS are of the order of 5\%,30\% and, respectively, $10 \%$ for $1.6 M_{\odot}$. Larger values, of the order of $10 \%, 60 \%$ and, respectively, $15 \%$ are obtained for more massive NS.

Finally, Fig. 8 plots the ratio and relative reduction of the maximum mass of hypernuclear stars with respect to the maximum mass of purely nucleonic stars. Possible correlations can be judged upon by considering the variety of 

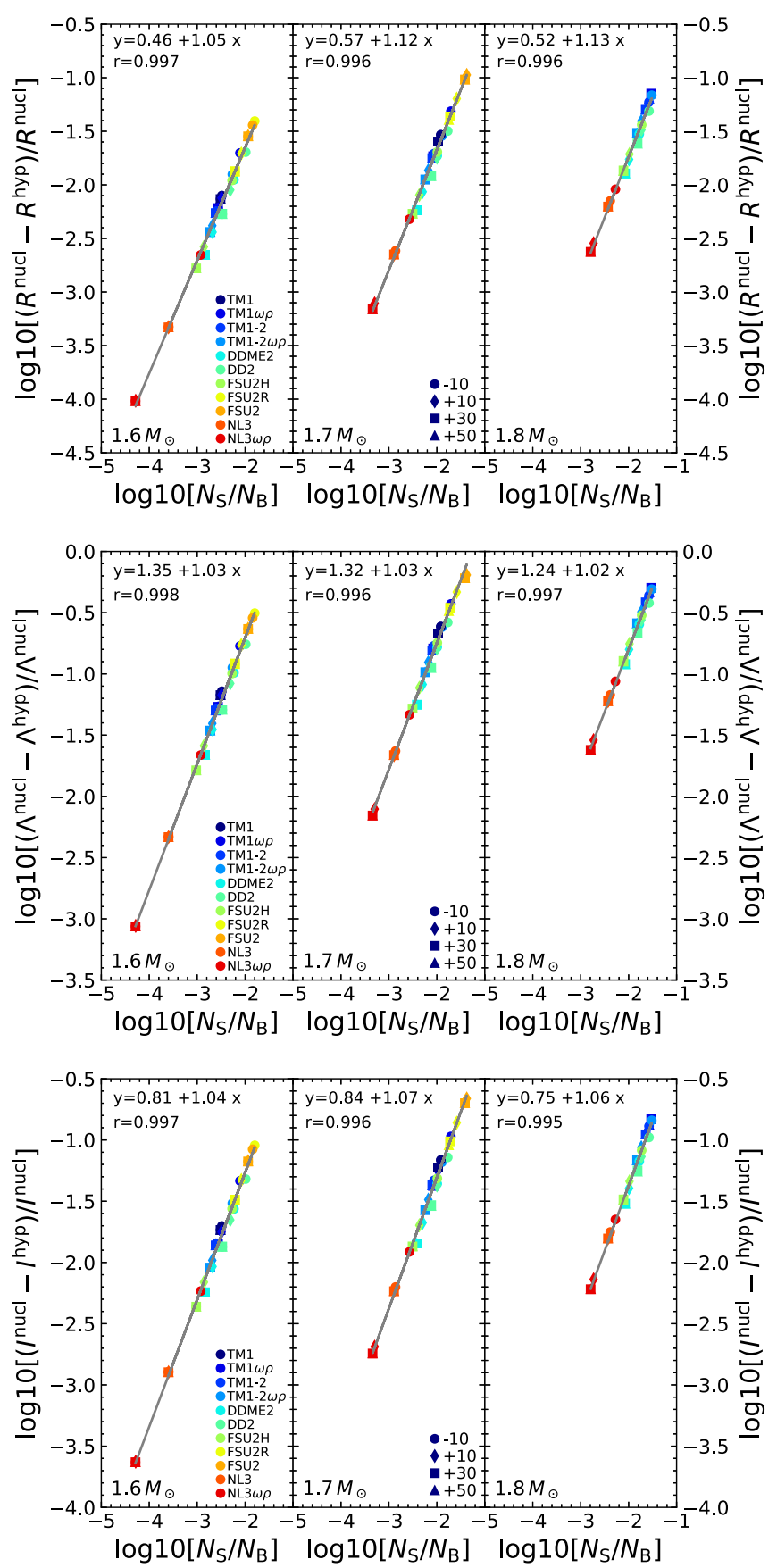

FIG. 7. Correlations between relative deviations of quantities that characterize hypernuclear stars from those that characterize purely nucleonic stars, and the strangeness fraction. Considered are the radius (top panels), the tidal deformability (middle panels) and the moment of inertia (bottom panels). The results correspond to various nucleonic EoS and $U_{\Sigma}^{(N)}$ values, as mentioned in the key legend. Results are for $1.6 M_{\odot}, 1.7 M_{\odot}$ and $1.8 M_{\odot}$. The correlation coefficient $r$ and the parameters of the linear fit are mentioned on each panel.

nucleonic EoS introduced in Sec. II A and a range of $U_{\Sigma}^{(N)}$ values. The first conclusion is that the inclusion of strangeness reduces the maximum mass by $\approx 15 \%-20 \%$, out of which $5 \%$ comes from insufficient knowledge on the

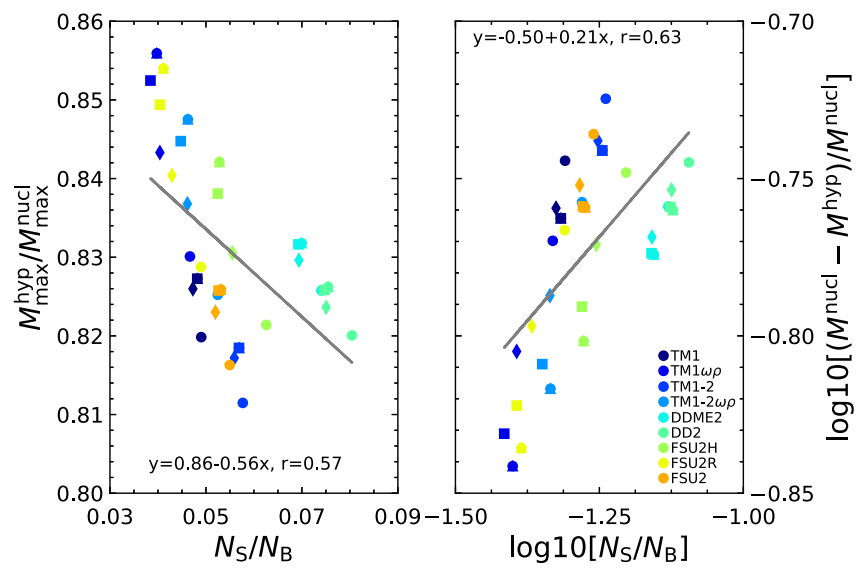

FIG. 8. Correlations between the strangeness fraction and the ratio (left panel) and, respectively, relative deviation (right panel) between maximum mass of hypernuclear stars and maximum mass of purely nucleonic stars. The same nucleonic EoS and values of $U_{\Sigma}^{(N)}$ as in Fig. 7 are considered. The correlation coefficients $r$ and the linear fit parameters are mentioned on each panel.

interaction between $\Sigma$-hyperons and nucleons. This means that, in order to produce hypernuclear stars with masses $\gtrsim 2 M_{\odot}$, the nucleonic EoS should be stiff enough to provide for purely nucleonic stars maximum masses larger than 2.35-2.5 $M_{\odot}$. Contrary to what we have seen in Fig. 7, only a loose correlation exists between $M_{\max }^{\text {hyp }} / M_{\max }^{\text {nucl }}$ and $N_{S} / N_{B}$ and $\log \left(1-M_{\max }^{\text {hyp }} / M_{\max }^{\text {nucl }}\right)$ and $\log \left(N_{S} / N_{B}\right)$. This result is easy to understand considering that no constraint is imposed on the high density behavior of the dominant nucleonic component, as no data exist so far in that region. As a consequence, properties of stars with quite different masses are being compared.

\section{CONCLUSIONS}

Starting from a set of nucleonic RMF models that explore widely different behaviors in the isoscalar and isovector channels and predict NS maximum masses in excess to the astrophysical $2 M_{\odot}$ constraint, we have studied the effect of including hyperons on the properties of NS. With the aim of constraining as much as possible the hyperon-nucleon interaction, we employ $\sigma Y$ coupling constants calibrated on experimental data on hypernuclei. The method relies on comparison between the values of the binding energy of nuclei with a variable number of nucleons and one or more hyperons, as obtained by solving the Dirac equation, with corresponding experimental data. More precisely, we employ the $g_{\sigma \Lambda}$ values determined in Refs. [8-10], based on a vast collection of data of single- $\Lambda$ hypernuclei, and further determine $g_{\sigma \Xi}$ from the binding energy of $\Xi^{-}$in the hypernucleus ${ }_{\Xi}^{15} \mathrm{C}$ [68]. Experimental uncertainties related to the final state of the daughter nucleus ${ }_{\Lambda}^{10} \mathrm{Be}$ are accounted for by considering the 
possibility that $\Xi^{-}$occupies the $1 s$ or, alternatively, the $1 p$ state of ${ }^{14} \mathrm{~N}$, as previously done in Ref. [63]. Uncertainties related to the $\Sigma N$ interaction are dealt with by allowing the $U_{\Sigma}^{(N)}$ potential to span a wide domain.

For all considered models and $U_{\Sigma}^{(N)}$ potentials, we have investigated NS geometric, deformability and chemical properties. Special attention was devoted to the density at which various hyperonic species nucleate and their sensitivity to the nucleonic EoS and $\Sigma N$ interaction. Dependence on these ingredients of the nucleonic and hyperonic dUrca thresholds was discussed as well. In regards to $\Sigma^{-}$we noticed that (a) in most NL models only two hyperon species are present: the $\Lambda$ and $\Sigma^{-}$. For the DD models the $\Xi^{-}$also appears. (b) The value of the $U_{\Sigma}^{(N)}$ determines the hyperonic dUrca process that opens up first, with a less (more) repulsive potential favoring the more (less) efficient $\Sigma \Lambda(\Lambda p)$ process [93]; (c) for repulsive values of $U_{\Sigma}^{(N)}$ potential, as customarily assumed in the literature, the $\Lambda p$ dUrca process starts operating at $n \approx 2 n_{0}$, which corresponds to $M / M_{\odot} \approx 1.3-1.4 M_{\odot}$. We have obtained very strong linear correlations between the strangeness fraction in the NS core and the relative deviation of the radius, tidal deformability and moment of inertia of hypernuclear compact stars from values characterizing purely nucleonic stars. Quantitatively speaking, for NS with masses in excess of $1.6 M_{\odot}$ hyperonic degrees of freedom are responsible for a reduction of radii, tidal deformabilities and moments of inertia of the order of $\sim 10 \%, 60 \%$ and, respectively, $15 \%$. In regards to the maximum mass, the decrease is of $15 \%$ to $20 \%$, with a non-negligible role played by $U_{\Sigma}^{(N)}$.

\section{ACKNOWLEDGMENTS}

This work was supported by Fundação para a Ciência e Tecnologia, Portugal, under Grants No. UID/FIS/04564/ 2019 and No. POCI-01-0145-FEDER-029912 with financial support from Science, Technology and Innovation, in its FEDER component, and by the FCT/MCTES budget through national funds (OE), and by the Polish National Science Centre (NCN) under Grant No. 2017/26/D/ST9/ 00591. A. R. R. acknowledges the support provided by the European COST Action "PHAROS" (Grant No. CA16214), through a STSM grant as well as the kind hospitality of the Department of Physics, University of Coimbra. C. P. acknowledges the support of THEIA networking activity of the Strong 2020 Project. This work was partially supported by Conselho Nacional de Desenvolvimento Científico e Tecnológico (CNPq) under Grant No. 6484/2016-1 (S. S. A.), and as a part of the project INCT-FNA (Instituto Nacional de Ciência e Tecnologia-Física Nuclear e Aplicações) No. 464898/ 2014-5 (S. S. A.).
[1] B. P. Abbott et al. (The Virgo, The LIGO Scientific Collaborations), Phys. Rev. Lett. 119, 161101 (2017).

[2] B. P. Abbott et al. (LIGO Scientific, Virgo, Fermi GBM, INTEGRAL, IceCube, AstroSat Cadmium Zinc Telluride Imager Team, IPN, Insight-Hxmt, ANTARES, Swift, AGILE Team, 1M2H Team, Dark Energy Camera GW-EM, DES, DLT40, GRAWITA, Fermi-LAT, ATCA, ASKAP, Las Cumbres Observatory Group, OzGrav, DWF (Deeper Wider Faster Program), AST3, CAASTRO, VINROUGE, MASTER, J-GEM, GROWTH, JAGWAR, CaltechNRAO, TTU-NRAO, NuSTAR, PanSTARRS, MAXI Team, TZAC Consortium, KU, Nordic Optical Telescope, ePESSTO, GROND, Texas Tech University, SALT Group, TOROS, BOOTES, MWA, CALET, IKI-GW Follow-up, H.E.S.S., LOFAR, LWA, HAWC, Pierre Auger, ALMA, Euro VLBI Team, Pi of Sky, Chandra Team at McGill University, DFN, ATLAS Telescopes, High Time Resolution Universe Survey, RIMAS, RATIR, SKA South Africa/MeerKAT), Astrophys. J. 848, L12 (2017).

[3] N. K. Glendenning, Compact stars: Nuclear Physics, Particle Physics, and General Relativity, 2nd ed. (SpringerVerlag, New York, 2000).
[4] A. Drago, A. Lavagno, G. Pagliara, and D. Pigato, Phys. Rev. C 90, 065809 (2014).

[5] B.-J. Cai, F. J. Fattoyev, B.-A. Li, and W. G. Newton, Phys. Rev. C 92, 015802 (2015).

[6] P. Ribes, A. Ramos, L. Tolos, C. Gonzalez-Boquera, and M. Centelles, Astrophys. J. 883, 168 (2019).

[7] J. J. Li and A. Sedrakian, Astrophys. J. 874, L22 (2019).

[8] M. Fortin, S. S. Avancini, C. Providência, and I. Vidaña, Phys. Rev. C 95, 065803 (2017).

[9] M. Fortin, M. Oertel, and C. Providência, Pub. Astron. Soc. Aust. 35, e044 (2018).

[10] C. Providência, M. Fortin, H. Pais, and A. Rabhi, Front. Astron. Space Sci. 6, 13 (2019).

[11] K. Nakazawa, Y. Endo, S. Fukunaga, K. Hoshino, S. H. Hwang, K. Imai, H. Ito, K. Itonaga, T. Kanda, M. Kawasaki et al., Prog. Theor. Exp. Phys. 2015, 033 D02 (2015).

[12] A. Gal, Prog. Theor. Phys. Suppl. 186, 270 (2010).

[13] A. Gal, E. V. Hungerford, and D. J. Millener, Rev. Mod. Phys. 88, 035004 (2016).

[14] H. Sugimura et al. (J-PARC E10 Collaboration), Phys. Lett. B 729, 39 (2014).

[15] R. Honda, M. Agnello, J. K. Ahn, S. Ajimura, Y. Akazawa, N. Amano, K. Aoki, H. C. Bhang, N. Chiga, M. Endo et al. 
(J-PARC E10 Collaboration), Phys. Rev. C 96, 014005 (2017).

[16] T. Harada, R. Honda, and Y. Hirabayashi, Phys. Rev. C 97, 024601 (2018).

[17] J. Haidenbauer and U.-G. Meißner, Nucl. Phys. A936, 29 (2015).

[18] P. Demorest, T. Pennucci, S. Ransom, M. Roberts, and J. Hessels, Nature (London) 467, 1081 (2010).

[19] Z. Arzoumanian et al. (NANOGrav Collaboration), Astrophys. J. Suppl. Ser. 235, 37 (2018).

[20] I. Vidana, D. Logoteta, C. Providencia, A. Polls, and I. Bombaci, Europhys. Lett. 94, 11002 (2011).

[21] D. Chatterjee and I. Vidaña, Eur. Phys. J. A 52, 29 (2016).

[22] I. Bednarek, P. Haensel, J. L. Zdunik, M. Bejger, and R. Mánka, Astron. Astrophys. 543, A157 (2012).

[23] S. Weissenborn, D. Chatterjee, and J. Schaffner-Bielich, Phys. Rev. C 85, 065802 (2012); 90, 019904(E) (2014).

[24] S. Weissenborn, D. Chatterjee, and J. Schaffner-Bielich, Nucl. Phys. A914, 421 (2013).

[25] G. Colucci and A. Sedrakian, Phys. Rev. C 87, 055806 (2013).

[26] E. N. E. van Dalen, G. Colucci, and A. Sedrakian, Phys. Lett. B 734, 383 (2014).

[27] M. Fortin, C. Providencia, A. R. Raduta, F. Gulminelli, J. L. Zdunik, P. Haensel, and M. Bejger, Phys. Rev. C 94, 035804 (2016).

[28] T.-T. Sun, S.-S. Zhang, Q.-L. Zhang, and C.-J. Xia, Phys. Rev. D 99, 023004 (2019).

[29] J. Antoniadis et al., Science 340, 1233232 (2013).

[30] H. T. Cromartie, E. Fonseca, S. M. Ransom et al., Nat. Astron. 4, 72 (2020).

[31] M. Alford, D. Blaschke, A. Drago, T. Klahn, G. Pagliara, and J. Schaffner-Bielich, Nature (London) 445, E7 (2007).

[32] S. Weissenborn, I. Sagert, G. Pagliara, M. Hempel, and J. Schaffner-Bielich, Astrophys. J. Lett. 740, L14 (2011).

[33] L. Bonanno and A. Sedrakian, Astron. Astrophys. 539, A16 (2012).

[34] K. Masuda, T. Hatsuda, and T. Takatsuka, Astrophys. J. 764, 12 (2013).

[35] M. G. Alford, S. Han, and M. Prakash, Phys. Rev. D 88, 083013 (2013).

[36] T. Klähn, R. Łastowiecki, and D. B. Blaschke, Phys. Rev. D 88, 085001 (2013).

[37] J. L. Zdunik and P. Haensel, Astron. Astrophys. 551, A61 (2013).

[38] D. Logoteta, C. Providência, and I. Vidaña, Phys. Rev. C 88, 055802 (2013).

[39] A. Drago, A. Lavagno, G. Pagliara, and D. Pigato, Eur. Phys. J. A 52, 40 (2016).

[40] R. C. Pereira, P. Costa, and C. Providência, Phys. Rev. D 94, 094001 (2016).

[41] K. Fukushima and T. Kojo, Astrophys. J. 817, 180 (2016).

[42] M. G. Alford and A. Sedrakian, Phys. Rev. Lett. 119, 161104 (2017).

[43] M. Dutra, O. Lourenço, S. S. Avancini, B. V. Carlson, A. Delfino, D. P. Menezes, C. Providência, S. Typel, and J. R. Stone, Phys. Rev. C 90, 055203 (2014).

[44] W.-C. Chen and J. Piekarewicz, Phys. Rev. C 90, 044305 (2014).
[45] L. Tolos, M. Centelles, and A. Ramos, Pub. Astron. Soc. Aust. 34, e065 (2017).

[46] R. Negreiros, L. Tolos, M. Centelles, A. Ramos, and V. Dexheimer, Astrophys. J. 863, 104 (2018).

[47] G. A. Lalazissis, J. Konig, and P. Ring, Phys. Rev. C 55, 540 (1997).

[48] H. Pais and C. Providência, Phys. Rev. C 94, 015808 (2016).

[49] C. J. Horowitz and J. Piekarewicz, Phys. Rev. Lett. 86, 5647 (2001).

[50] Y. Sugahara and H. Toki, Nucl. Phys. A579, 557 (1994).

[51] C. Providencia and A. Rabhi, Phys. Rev. C 87, 055801 (2013).

[52] S. S. Bao and H. Shen, Phys. Rev. C 89, 045807 (2014).

[53] S. Typel, G. Röpke, T. Klähn, D. Blaschke, and H. Wolter, Phys. Rev. C 81, 015803 (2010).

[54] G. A. Lalazissis, T. Nikšić, D. Vretenar, and P. Ring, Phys. Rev. C 71, 024312 (2005).

[55] S. Shlomo, V. M. Kolomietz, and G. Colo, Eur. Phys. J. A 30, 23 (2006).

[56] J. N. De, S. K. Samaddar, and B. K. Agrawal, Phys. Rev. C 92, 014304 (2015).

[57] J. M. Lattimer and Y. Lim, Astrophys. J. 771, 51 (2013).

[58] M. Oertel, M. Hempel, T. Klähn, and S. Typel, Rev. Mod. Phys. 89, 015007 (2017).

[59] S. Abrahamyan, Z. Ahmed, H. Albataineh, K. Aniol, D. S. Armstrong, W. Armstrong, T. Averett, B. Babineau, A. Barbieri, V. Bellini et al. (PREX Collaboration), Phys. Rev. Lett. 108, 112502 (2012).

[60] M. D. Cozma, Eur. Phys. J. A 54, 40 (2018).

[61] H. Shen, F. Yang, and H. Toki, Prog. Theor. Phys. 115, 325 (2006).

[62] S. S. Avancini, J. R. Marinelli, D. P. Menezes, M. M. W. de Moraes, and C. Providencia, Phys. Rev. C 75, 055805 (2007).

[63] T. T. Sun, E. Hiyama, H. Sagawa, H. J. Schulze, and J. Meng, Phys. Rev. C 94, 064319 (2016).

[64] J. Mares and J. Zofka, Z. Phys. A 333, 209 (1989).

[65] B. K. Jennings, Phys. Lett. B 246, 325 (1990).

[66] Y. Sugahara and H. Toki, Prog. Theor. Phys. 92, 803 (1994).

[67] J. Mareš and B. K. Jennings, Phys. Rev. C 49, 2472 (1994).

[68] P. Khaustov et al. (AGS E885 Collaboration), Phys. Rev. C 61, 054603 (2000).

[69] J. Yoshida et al., J. Phys. Soc. Jpn. Conf. Proc. 26, 023006 (2019).

[70] E. Hiyama, Y. Yamamoto, T. Motoba, T. A. Rijken, and M. Kamimura, Phys. Rev. C 78, 054316 (2008).

[71] H. Matsumiya, K. Tsubakihara, M. Kimura, A. Dote, and A. Ohnishi, Phys. Rev. C 83, 024312 (2011).

[72] E. Hiyama and Y. Yamamoto, Prog. Theor. Phys. 128, 105 (2012).

[73] D. Millener, Nucl. Phys. A881, 298 (2012).

[74] T. Gogami, C. Chen, D. Kawama, P. Achenbach, A. Ahmidouch, I. Albayrak, D. Androic, A. Asaturyan, R. Asaturyan, O. Ates et al. (HKS(JLab E05-115) Collaboration), Phys. Rev. C 93, 034314 (2016).

[75] N. K. Glendenning and S. A. Moszkowski, Phys. Rev. Lett. 67, 2414 (1991). 
[76] See Supplemental Material at http://link.aps.org/ supplemental/10.1103/PhysRevD.101.034017, for tables of all the unified equations of state used in this work: FSU2, FSU2H, FSU2R, NL3, NL3 $\omega \rho$, TM1, TM1 $\omega \rho$,

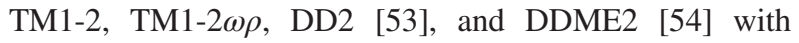
$U_{\Sigma}^{(N)}=-10,10,30$, and $50 \mathrm{MeV}$.

[77] R. C. Tolman, Phys. Rev. 55, 364 (1939).

[78] J. R. Oppenheimer and G. M. Volkoff, Phys. Rev. 55, 374 (1939).

[79] T. E. Riley, A. L. Watts, S. Bogdanov, P.S. Ray, R. M. Ludlam, S. Guillot, Z. Arzoumanian, C. L. Baker, A. V. Bilous, D. Chakrabarty et al., Astrophys. J. 887, L21 (2019).

[80] M. C. Miller, F. K. Lamb, A. J. Dittmann, S. Bogdanov, Z. Arzoumanian, K. C. Gendreau, S. Guillot, A. K. Harding, W. C. G. Ho, J. M. Lattimer et al., Astrophys. J. 887, L24 (2019).

[81] M. C. Miller and F. K. Lamb, Eur. Phys. J. A 52, 63 (2016).

[82] P. Haensel, M. Bejger, M. Fortin, and L. Zdunik, Eur. Phys. J. A 52, 59 (2016).

[83] Z. Arzoumanian, K. C. Gendreau, C. L. Baker, T. Cazeau, P. Hestnes, J. W. Kellogg, S. J. Kenyon, R. P. Kozon, K. C. Liu, S. S. Manthripragada et al., Proc. SPIE, 9144, 914420 (2014).
[84] C. Motch, J. Wilms, D. Barret, W. Becker, S. Bogdanov, L. Boirin, S. Corbel, E. Cackett, S. Campana, D. de Martino et al., arXiv:1306.2334.

[85] A. L. Watts, W. Yu, J. Poutanen, S. Zhang, S. Bhattacharyya, S. Bogdanov, L. Ji, A. Patruno, T. E. Riley, P. Bakala et al., Sci. China Phys. Mech. Astron. 62, 29503 (2019).

[86] J. B. Hartle, Astrophys. J. 150, 1005 (1967).

[87] M. Bejger, T. Bulik, and P. Haensel, Mon. Not. R. Astron. Soc. 364, 635 (2005).

[88] T. Hinderer, Astrophys. J. 677, 1216 (2008).

[89] B. P. Abbott, R. Abbott, T. D. Abbott, F. Acernese, K. Ackley, C. Adams, T. Adams, P. Addesso, R. X. Adhikari, V. B. Adya et al., Phys. Rev. Lett. 121, 161101 (2018).

[90] B. P. Abbott, R. Abbott, T. D. Abbott, F. Acernese, K. Ackley, C. Adams, T. Adams, P. Addesso, R. X. Adhikari, V. B. Adya et al., Phys. Rev. X 9, 011001 (2019).

[91] A. Watts et al., Proc. Sci. AASKA14 (2015) 043 [arXiv:1501.00042].

[92] J. M. Lattimer, C. J. Pethick, M. Prakash, and P. Haensel, Phys. Rev. Lett. 66, 2701 (1991).

[93] M. Prakash, M. Prakash, J. M. Lattimer, and C. J. Pethick, Astrophys. J. Lett. 390, L77 (1992). 